\title{
Inheritance and linkage of isozymes in Yponomeuta padellus (Lepidoptera, Yponomeutidae)
}

\author{
LÉON E. L. RAIJMANN* $\dagger$, WIL E. VAN GINKEL $\uparrow$, DAVID G. HECKEL $\ddagger$ \\ \& STEPH B. J. MENKEN† \\ $\dagger$ Institute for Systematics and Population Biology, University of Amsterdam, PO Box 94766, 1090 GT Amsterdam, \\ The Netherlands and łDepartment of Biological Sciences, Clemson University, Clemson, SC 29634-1903, U.S.A.
}

\begin{abstract}
Inheritance and genetic linkage of 29 allozyme loci were studied by single-pair crosses of Yponomeuta padellus (Lepidoptera, Yponomeutidae). All loci segregated as Mendelian genes with codominant alleles except for a null allele at the $h b d h$ locus. The three loci est-2, $6 \mathrm{pgdh}$, and fudh were sex-linked and occurred in that order along the $\mathrm{Z}$ chromosome. Autosomal linkage analysis was facilitated by the lack of crossing-over in females characteristic of Lepidoptera, because linkage in female-informative crosses is all-or-none and observation of 'forbidden recombinants' provides conclusive evidence that two loci are not syntenic. Convincing evidence was found for linkage of the autosomal loci me and mpi. This linkage group and $a a t-1, c a, h b d h, h k-1, p g i$ and pgm were assigned to seven separate autosomes. Weaker evidence from male-informative families supported four additional linked pairs. Previous cytological studies have shown that the sex chromosomes in heterogametic females are associated in a trivalent, consisting of a $\mathrm{W}$ chromosome translocated to an autosome $\left(\mathrm{A}^{\mathrm{w}}\right)$, paired with the $\mathrm{Z}$ chromosome and the homologous autosome $\mathrm{A}$. Any locus located on this autosome and its homologous segment on the $\mathrm{A}^{\mathrm{w}}$ chromosome should show strict segregation of one allele to sons and the other to daughters. However, none of the female-informative loci in this study satisfied that criterion. Use of the method of forbidden recombinants and its utility in the study of chromosomal evolution in Yponomeuta are discussed.
\end{abstract}

Keywords: achiasmatic meiosis, linkage, mapping of allozymes, sex chromosome trivalent.

\section{Introduction}

The genus Yponomeuta (Lepidoptera, Yponomeutidae) or small ermine moths has been used as a model system to study the evolution of insect-plant relationships (Menken et al., 1992; and references therein). Allozyme variation has been analysed intensively in host-associated populations of the oligophagous Y. padellus (Menken, 1982; Raijmann, 1996), in particular in relation to the study of host race formation (Raijmann \& Menken, 1992). Although there is extensive information about population genetics based on allozyme data in Y. padellus, information on the transmission genetics of allozymes in this species is still absent. Thus far, two studies have been carried out to study the inheritance of allozyme markers in Yponomeuta. First, the inheritance of five mainly diagnostic loci has been reported within the so-called 'padellus-complex', a

\footnotetext{
*Correspondence. E-mail: raijmann@bio.uva.nl
}

group of five morphologically similar species including Y. padellus (Menken, 1980a). Secondly, Menken (1980b) made an in-depth study of the inheritance of 10 enzyme systems in $Y$. cagnagellus. This paper is the first of a series of such studies in Y. padellus.

We have three main goals in this work. The first is to establish which allozymes are sex-linked in $Y$. padellus. In a recent review, Sperling (1994) pointed out that in many groups of Lepidoptera, characteristics affecting reproductive isolation and host race formation appear to be predominantly sex-linked. However, he identified Yponomeuta as a group to which this generalization did not apply (see Van Drongelen \& Van Loon, 1980; Hendrikse, 1988). To investigate further the validity of these claims, we would like to identify as many sex-linked marker loci as possible.

Our second goal is to begin construction of a linkage map for the autosomes of $Y$. padellus. Comparative linkage mapping in Lepidoptera is in its infancy compared to genome mapping in 
mammals (O'Brien et al., 1988) and in certain plant families (Bennetzen \& Freeling, 1993), but mapping a common set of anchor loci such as allozymes is a reasonable first step towards that goal (Heckel, 1993). The general lack of crossing-over in female Lepidoptera (Robinson, 1971) greatly facilitates autosomal linkage mapping because syntenic pairs of loci produce no recombinants in female-informative crosses: linkage, being all-or-none in such crosses, is easy to detect or to rule out.

Our third goal is to seek genetic evidence to corroborate cytogenetic investigations in the genus. Nilsson et al. (1988) studied the first meiotic division in males and females of several Yponomeuta species, including $Y$. padellus. They found that the sex chromosomes in the heterogametic females were associated in a sex chromosome trivalent (written as $\mathrm{AA}^{\mathrm{w}} \mathrm{Z}$ ), consisting of a $\mathrm{W}$ chromosome translocated to an autosome to form an $\mathrm{A}^{\mathrm{w}}$ chromosome. This $\mathrm{A}^{\mathrm{w}}$ chromosome was paired with the homologous autosome and the $\mathrm{Z}$ chromosome. In the homogametic males the two $\mathrm{Z}$ chromosomes were paired in the usual bivalent, thus the male karyotype could be represented as $2 n=30 \mathrm{AA}+\mathrm{ZZ}$, and the female karyotype as $2 n=29 \mathrm{AA}+\mathrm{AA}^{\mathrm{w}} \mathrm{Z}$. Transmission genetics studies would give us the opportunity to test allozyme loci for their occurrence on the translocated autosome.

In this paper, single-pair crosses of $Y$. padellus were used to analyse the inheritance of 22 enzyme systems in support of these three objectives. The high level of genetic polymorphism in the source population provided enough variability to address our questions, without the need of first creating inbred strains. Our results to date suggest specific strategies for building on this information, to exploit the favourable features of the genetic system of Lepidoptera in comparative genome studies within Yponomeuta, and to investigate the evolutionary dynamics of host race formation.

\section{Materials and methods}

Over 300 fifth instar larvae were collected from a $Y$. padellus population infesting Crataegus spp. in Leiden, The Netherlands. These larvae were raised to adulthood in the laboratory and undamaged specimens were selected for the breeding experiments. Immediately after emergence, one male and one female were placed in a glass jar together with a young twig of Crataegus spp. as oviposition substrate, and an eppendorf tube with sugar-water (5 per cent $\mathrm{w} / \mathrm{v}$ glucose) as a substitute for nectar. A total of 51 such single-pair crosses was set up.

\section{Electrophoresis and enzyme staining}

Sample preparations and starch gel electrophoresis were carried out between $0^{\circ} \mathrm{C}$ and $4^{\circ} \mathrm{C}$. Wings and legs were removed, and individual moths were homogenized in $125 \mu \mathrm{L}$ of grinding buffer containing $10 \mathrm{~mm}$ Tris, $10 \mathrm{~mm}$ maleic acid, $1 \mathrm{~mm}$ EDTA, $1 \mathrm{~mm}$ $\mathrm{MgCl}_{2}, 0.05 \mathrm{~mm}$ NADP and $\mathrm{pH}$ adjusted to 7.4 with $\mathrm{NaOH}$. Extracts were centrifuged at $9615 \mathrm{~g}$ at $4^{\circ} \mathrm{C}$ for $5 \mathrm{~min}$ and the clear supernatant was either stamped on cellulose acetate gels or soaked onto Whatman 3 MM chromatography paper wicks for insertion into 11 per cent w/v Connaught starch gels. To analyse fumarate hydratase and sucrase, we carried out cellulose acetate electrophoresis following the procedure of Hebert \& Beaton (1989), using Titan III cellulose acetate plates (Helena Laboratories, Beaumont, TX, U.S.A.). All other enzymes were studied on starch gels, prepared according to Ayala et al. (1972). The clarity of both phosphoglucomutase and 6-phosphogluconic dehydrogenase was much improved by adding $0.06 \mathrm{mM}$ NADP to the heated gel mixture and in the cathode vessel prior to electrophoresis. Buffer systems, staining methods, subunit structure, and number of alleles for all enzyme systems are listed in Table 1 . Sucrase was stained with a solution of $0.2 \mathrm{M} \mathrm{Na} \mathrm{NPO}_{4}$ adjusted with citric acid to $\mathrm{pH}=7.0,15 \mathrm{mg} \mathrm{mL}^{-1}$ sucrose, $1 \mathrm{mg} \mathrm{mL}^{-1}$ glucose oxidase, $1.5 \mathrm{mg} \mathrm{mL}^{-1} \mathrm{MTT}$ and $0.5 \mathrm{mg} \mathrm{mL}^{-1}$ PMS with a 1 per cent agar overlay. Multiple loci were numbered sequentially from the most cathodally migrating protein product. Allele designation generally follows Menken (1982) and Arduino \& Bullini (1985).

\section{Linkage analysis}

For the initial steps in data analysis, we used version 3.50 of the LINKAGE-1 program (Suiter et al., 1983). The error-checking and equal-segregation tests of LINKAGE-1 are appropriate to our data, but its method of estimating recombination rates depends critically on the assumption of crossing-over in both sexes, which is not valid here. LINKAGE-1 also makes certain assumptions about gametic phase which may be too strong when phase is unknown. Thus for analysing linkage, we used only part of the LINKAGE-1 output, as described below. Estimation of recombination fractions among the sex-linked loci was straightforward, because hemizygosity of females rendered the situation formally equivalent to a backcross. The remaining analysis was restricted to autosomal loci.

The LINKAGE-1 printout was examined by hand, to separate the locus pairs into four types: informative 


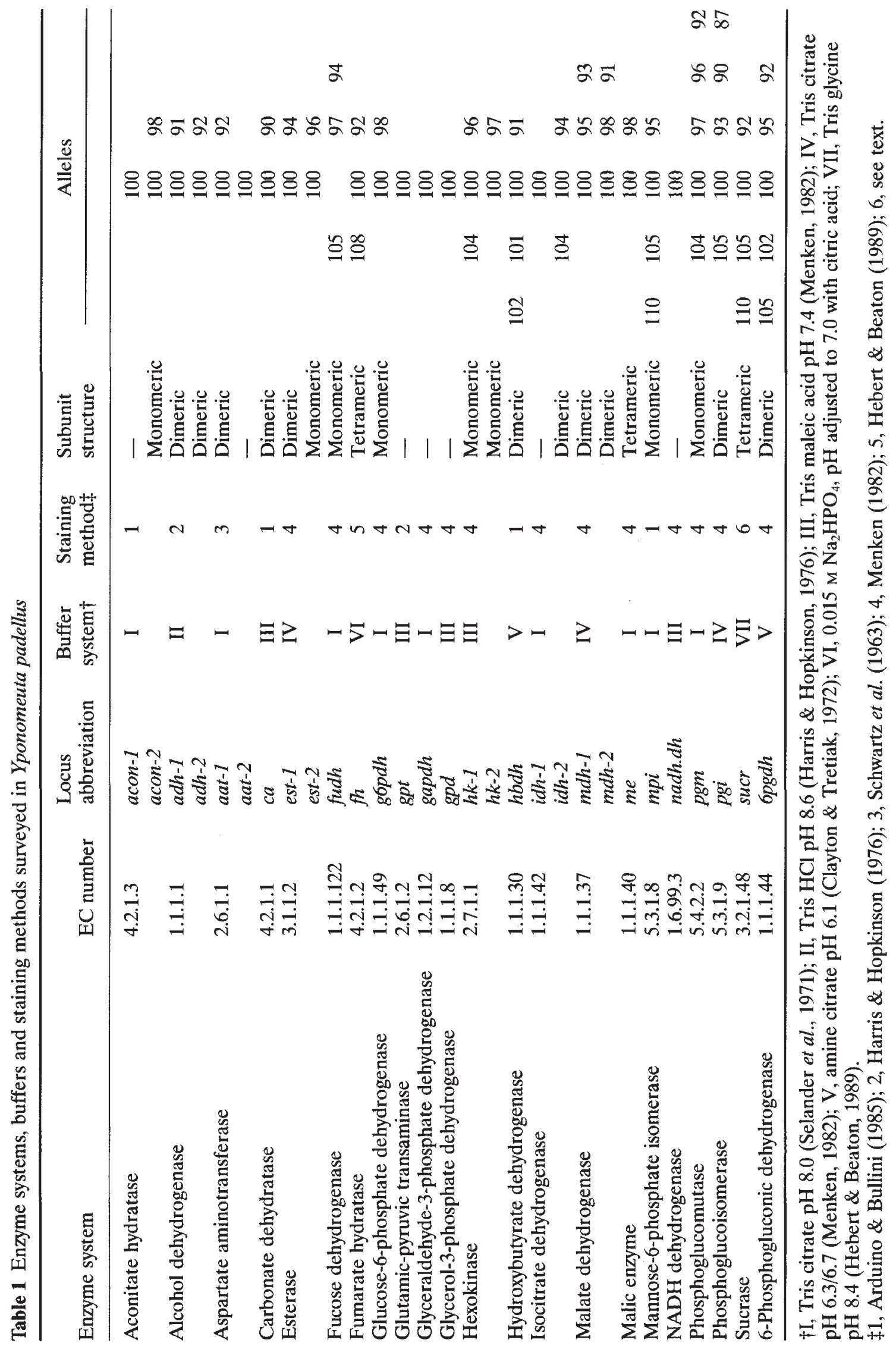


in females only, informative in males only, informative in both parents, and informative in neither parent. (A locus pair is informative for linkage in a parent if, and only if, that parent is heterozygous for both loci.) Within each of the first three types, data from all crosses were tabulated by locus pair. For those crosses of the third type with three or more alleles entering at one or both loci, recombinations in males and females could be clearly distinguished.

Because females show no crossing-over in Yponomeuta (Nilsson et al., 1988), as in other Lepidoptera (Robinson, 1971), data from the tabulated locus pairs informative in mothers (types 1 and 3) were examined for forbidden recombinants. These are defined as certain offspring genotypes that could not be produced if the two loci were syntenic. Because the gametic phase is unknown in these crosses, both phase possibilities must be examined; if both produce forbidden recombinants, the two loci are asyntenic (they must occupy separate chromosomes). Let $F R_{\min }$ be the smaller of the two numbers of observed forbidden recombinants, each calculated under a different assumption of phase for a specific cross. $F R_{\min } \geq 1$ corresponds to asynteny, the case just mentioned. However, if $F R_{\min }=0$, synteny is not ruled out by this cross. $F R_{\min }=0$ could also be observed by chance in small progenies, but if data from several crosses informative for the same pair of loci are available, and if the sum of $F R_{\min }$ over crosses is one or more, the two loci must be asyntenic. When $F R_{\min }$ was zero for all families, significance of linkage was assessed using the lod score method (Ott, 1991). Because counting forbidden recombinants is sensitive to scoring errors, locus pairs with summed $F R_{\text {min }}=1$ were re-examined for this possibility.

Data from the tabulated locus pairs informative in fathers only (type 2) were examined for deviation from independence using the test provided by LINKAGE-1. Because crossing-over occurs in males, the method of forbidden recombinants does not apply and linkage detection proceeds in the conventional manner. The program tests for linkage by calculating the $\chi^{2}$-value associated with the tabulated genotypes, under the null hypothesis of independent assortment. Linkage is declared when a significant $\chi^{2}$-value $(P<0.05)$ is found for a given locus pair. Tight linkage would produce an excess of certain genotypes and a deficiency of others detectable by this test. However, the hypothesis of independence can be rejected for reasons other than linkage, especially when three or more genotypes occur at each locus. It can even be rejected when the cross is not informative, resulting in a spurious declaration of linkage by the program. Thus we re-examined each case where LINKAGE-1 declared linkage, for confirmation.

Each locus pair for which LiNKAGE-1 rejected independent assortment at $P<0.05$ for at least one maleinformative family, and for which there was not evidence for asynteny from female-informative families, was re-evaluated using the method of lod scores (Ott, 1991). In all but one of these cases, the male was the only informative parent, facilitating calculation of the $Z$-values. In the other case, some crosses had both parents informative, but the presence of three or four alleles at one of the loci enabled unambiguous identification of male vs. female recombination.

To examine female-informative loci for possible association with the $\mathrm{A}^{\mathrm{w}}$ translocation, allelic segregation was examined in relation to the sex of offspring. Sex was coded as $z z$ for males and $z w$ for females in the LINKAGE-1 dataset, corresponding to the chromosomal sex determination mechanism. The method of forbidden recombinants described above was employed using this 'sex locus' and all allozyme loci heterozygous in one or more mothers. If the allozyme was located on the translocated autosome and its unattached homologue, one allele should be transmitted exclusively to daughters along with the attached $W$ and the other to sons along with the unattached Z. As gametic phase was unknown, both possibilities were considered, the value of $F R_{\min }$ was calculated and these were summed over all crosses, informative for linkage to the same allozyme.

\section{Results}

\section{Inheritance of allozymes}

The 22 different enzyme systems comprised a total of 29 presumptive loci (Table 1). Seven enzyme systems, ACON-1, AAT-2, GPT, GAPDH, GPD, IDH-1 and NADH.DH were encoded by monomorphic loci.

Two crosses did not fit Mendelian expectations of equal segregation, among a total of 98 tests of autosomal genes. Cross 8 produced a deficiency of heterozygotes at est-1 $\left(\chi_{1}^{2}=11.8, P=0.0006\right)$, and cross 15 an excess of heterozygotes at adh-1 $\left(\chi_{2}^{2}=7.6, P=0.02\right)$. We attribute both deviations from Mendelian inheritance to nonbiological factors in view of the fact that the number of rejections is quite small (the Type I error is less than 5 per cent).

All loci encoding allozymes displayed exclusively codominant alleles in this study except $h b d h$. The dimer hydroxybutyrate dehydrogenase usually displays the typical three-banded pattern in hetero- 
zygotes, with the middle band staining about twice as intensely as the other two. However, a two-banded electromorph with equal staining intensity in the two bands was also seen in these crosses, as in earlier population surveys. These phenotypes appeared to be heterozygotes for a null allele, in which the protein product had undetectable enzyme activity but formed active dimers with the polypeptide of the other allele. Five of the crosses had a parent with this phenotype (one male and four females), and in each case the offspring phenotypes indicated Mendelian transmission of the null allele.

\section{Sex-linkage}

Fudh, est-1 and 6pgdh showed transmission patterns characteristic of sex-linked loci in Lepidoptera, with hemizygous mothers transmitting their only allele always and exclusively to sons, and heterozygous fathers segregating their two alleles equally to offspring irrespective of sex (Table 2).

However, two apparent females were scored as heterozygotes (one offspring of cross 3 for fudh, and one offspring of cross 10 for $6 p g d h$ ), expressing an allele identical to their mother's as well as the expected paternal allele (Table 2). Such heterozygotes would be expected only among male progeny of these crosses. Conversely, in other crosses, three apparent male offspring were scored as $6 p g d h$ homozygotes of a type that would be expected only among hemizygous female progeny. In cross 23 the phenotypes of two sons were 105/105, although their father's phenotype was $105 / 100$ and their mother's was $100 \%$. In cross 21 the electromorph pattern of one son was $102 / 102$, although his father's was $102 / 100$ and his mother's was $95 /$ (Table 2). In all other offspring of these crosses, sexes corresponded to genotypes expected at sex-linked loci. Furthermore, for none of the autosomally encoded loci informative in these same crosses was there any inconsistencies between parental and offspring genotypes. We believe that these anomalous individuals may actually be gynandromorphs (see Discussion). To avoid the possibility of spurious results, the five anomalous individuals were excluded from all subsequent linkage calculations.

Crosses 3, 5, 13, 21 and 23 were informative for linkage among the sex-linked loci. Assuming that the most likely phase in each cross is the true phase, there was a total of $5 / 39=12.8$ per cent recombi-

Table 2 Segregation data of three sex-linked allozymes in Yponomeuta padellus. Electromorph patterns are given for the male and female parents, and for the progeny with number of individuals scored, respectively

\begin{tabular}{|c|c|c|c|c|c|c|c|c|c|}
\hline \multirow[b]{2}{*}{ Locus } & \multirow[b]{2}{*}{ Cross (no.) } & \multirow[b]{2}{*}{$\mathrm{P}_{0} \hat{x}$} & \multirow[b]{2}{*}{ Po } & \multicolumn{6}{|c|}{$F_{1}$-genotype numbers } \\
\hline & & & & & o & & & $q$ & \\
\hline \multirow[t]{4}{*}{ est-2 } & 2 & $96 / 100$ & $100 /$ & 96/100:6 & 100/100:1 & & $96 /: 1$ & $100 /: 4$ & \\
\hline & 5 & $96 / 100$ & $100 /$ & $96 / 100: 8$ & $100 / 100: 8$ & & $96 /: 8$ & $100 /: 8$ & \\
\hline & 23 & $96 / 100$ & $100 /$ & 100/100:4 & & & $96 /: 2$ & $100 /: 3$ & \\
\hline & $8,10,13,15,18,19,20,21$ & $100 / 100$ & $100 /$ & 100/100:69 & & & $100 /: 72$ & & \\
\hline \multirow[t]{5}{*}{ fudh } & 3 & $97 / 104$ & $97 /$ & $97 / 97: 5$ & 97/104:8 & & $97 /: 8$ & $104 /: 9$ & 97/104:1 \\
\hline & 13 & 94/97 & $97 /$ & 94/97:5 & 97/97:5 & & $94 /: 3$ & $97 /: 5$ & \\
\hline & 21 & $97 / 104$ & $97 /$ & 97/97:7 & 97/104:7 & & $97 /: 8$ & 104/:9 & \\
\hline & 23 & $97 / 104$ & $97 /$ & 97/97:2 & 97/104:2 & & $97 /: 2$ & $104 /: 3$ & \\
\hline & $5,8,9,10,15,19,20$ & 97/97 & $97 /$ & 97/97:66 & & & $97 /: 75$ & & \\
\hline \multirow{13}{*}{$6 p g d h$} & 2 & $102 / 102$ & $100 /$ & 102/100:7 & & & $102 /: 5$ & & \\
\hline & 3 & $105 / 95$ & $95 /$ & 105/95:7 & 95/95:6 & & $105 /: 9$ & $95 /: 9$ & \\
\hline & 5 & $100 / 95$ & $95 /$ & $100 / 95: 7$ & 95/95:9 & & $100 /: 10$ & $95 /: 6$ & \\
\hline & 8 & $100 / 95$ & $102 /$ & 102/100:4 & $102 / 95: 3$ & & $100 /: 5$ & $95 /: 7$ & \\
\hline & 9 & $95 / 92$ & $95 /$ & 95/95:4 & 95/92:4 & & $95 /: 7$ & $92 /: 8$ & \\
\hline & 10 & $105 / 102$ & $100 /$ & 105/100:1 & $102 / 100: 4$ & & $105 /: 3$ & $102 /: 6$ & $102 / 100: 1$ \\
\hline & 13 & $105 / 102$ & $100 /$ & 105/100:4 & 102/100:6 & & $105 /: 5$ & $102 /: 3$ & \\
\hline & 15 & $102 / 100$ & $105 /$ & 105/102:2 & $105 / 100: 3$ & & $102 /: 4$ & $100 /: 1$ & \\
\hline & 18 & $100 / 100$ & $105 /$ & 105/100:3 & & & $100 /: 3$ & & \\
\hline & 19 & $105 / 105$ & $105 /$ & 105/105:11 & & & $105 /: 8$ & & \\
\hline & 20 & $100 / 95$ & $100 /$ & 100/100:7 & $100 / 95: 5$ & & $100 /: 8$ & $95 /: 1$ & \\
\hline & 21 & $102 / 100$ & $95 /$ & 102/102:1 & $102 / 95: 7$ & 100/95:6 & $102 /: 6$ & $100 /: 11$ & \\
\hline & 23 & $105 / 100$ & $100 /$ & 105/105:2 & 105/100:2 & & $105 /: 3$ & $100 /: 2$ & \\
\hline
\end{tabular}


nants between est- 2 and $6 p g d h, 15 / 85=17.6$ per cent recombinants between $6 p g d h$ and fudh, and $2 / 7=28.6$ per cent recombinants between est- 2 and fudh. Based upon the data of these 131 offspring a provisional map of the $\mathrm{Z}$ chromosome can be constructed (Table 3 ). A similar result was found in Heliothis virescens: within this species there is about 30 per cent recombination between fudh and $6 \mathrm{pgdh}$ (D. G. Heckel, unpublished data).

All other polymorphic loci showed widespread occurrence of heterozygotes among females, comparable to the frequencies in males, conclusively establishing them as autosomally encoded.

\section{Genetic linkage analysis of autosomal loci}

Two loci (acon-2 and est-1) were heterozygous in mothers only, three $(f h, i d h-2$ and $m d h-1)$ were heterozygous in fathers only, and the remaining 14 (aat-1, adh-1, adh-2, ca, g6pdh, hbdh, hk-1, hk-2, $m d h-2, m e, m p i, p g i, p g m$ and sucr) were heterozygous in both mothers and fathers. This restricted the number and type of linkage tests that could be carried out with the present data; e.g. linkage of $f$ h to other loci could not be examined by the method of forbidden recombinants because no mothers were heterozygous for $f h$. The number of linkage tests was further restricted by the numbers of mothers or fathers heterozygous for two or more loci simultaneously.

\section{Female-informative loci}

Of the 120 possible pairwise tests among the 16 loci heterozygous in mothers, 65 were informative in the

Table 3 Linkage groups of allozyme loci in Yponomeuta padellus. Map distances are recombination percentages

\section{Linkage groups}

1 Sex-chromosome:

\begin{tabular}{lll}
\multicolumn{3}{c}{28.6} \\
\end{tabular}

dataset for one or more families (Table 4). Fifty-six pairwise tests had summed $F R_{\min }$ values $>1$ and are good evidence of asynteny, six pairs had summed $F R_{\min }=1$, and three pairs had summed $F R_{\min }=0$ consistent with synteny. The latter three pairs are $a d h-1 / h k-1, a d h-1 / m p i$, and me/mpi. The pair me/mpi showed no forbidden recombinants among 50 progeny, and their linkage is also supported by maleinformative families (see below). However, one or both of the pairs $a d h-1 / h k-1$ and $a d h-1 / m p i$ must be unlinked because otherwise the locus group adh-1/mpi/me/hk-1 would be syntenic, and this is ruled out by the presence of forbidden recombinants in the $m p i / h k-1$ comparison (Table 4). Moreover, the progeny totals for adh-1/hk-1 and adh-1/mpi femaleinformative families are very low, and there are no male-informative families for either locus pair to test further these linkage groups. Gels for the six locus pairs with summed $F R_{\text {min }}=1$ were re-examined for possible scoring errors, but none was found. Low $F R_{\min }$ values for these pairs also probably result from the very small number of informative progeny for these combinations (Table 4).

The summed $F R_{\min }$ values show that at least seven separate chromosomes are marked by autosomal loci; i.e. $(m p i+m e)$, aat-1, ca, hbdh, hk-1, pgi and pgm must occupy separate autosomes (Table 3). This statement is possible because the dataset is informative for every possible pairwise comparison of these seven groups and many forbidden recombinants are found for each pairwise comparison (Table 4). Incompleteness of data limits the statements that can be made about the other loci, but at least seven but no more than 15 autosomes are marked by the 16 female-informative autosomal loci.

\section{Male-informative loci}

Of the 136 possible pairwise tests among the 17 loci heterozygous in fathers, 82 were informative in one or more families. Unfortunately, because crossingover occurs in males, the method of forbidden recombinants for ruling out synteny does not apply. However, strong deviation from independent assortment can provide evidence for linkage. LiNKAGE-1 examined 139 male-informative two-way tables for deviations from independent assortment, and reported 15 to be significant at $P<0.05$. Upon further investigation, some of these provided support for linkage.

The most striking deviation from independent assortment was the pair me/mpi, which was significant in all five male-informative families. Assuming the most likely phase configuration, there was only

(C) The Genetical Society of Great Britain, Heredity, 78, 645-654. 
Table $4 F R_{\min }$, the minimum number of forbidden recombinants, summed over all crosses informative in females and tabulated by locus pair. A dash (-) indicates that no female-informative crosses were present in the dataset for that locus pair. Total numbers of progeny for pairs in which zero or one forbidden recombinant was observed are as follows: $F R_{\min }=0:$ me/mpi (50), adh-1/mpi (8) adh-1/hk-1 (10); $F R_{\min }=1:$ sucr/mpi (9), mdh-2/mpi (19), adh-1/me (10), adh-1/pgi (10), adh-2/mpi (17), adh-2/adh-1 (8)

\begin{tabular}{|c|c|c|c|c|c|c|c|c|c|c|c|c|c|c|c|c|c|}
\hline & & 1 & 2 & 3 & 4 & 5 & 6 & 7 & 8 & 9 & 10 & 11 & 12 & 13 & 14 & 15 & 16 \\
\hline 1 & me & & & & & & & & & & & & & & & & \\
\hline 2 & $m p i$ & 0 & & & & & & & & & & & & & & & \\
\hline 3 & $p g m$ & 9 & 44 & & & & & & & & & & & & & & \\
\hline 4 & $h b d h$ & 17 & 27 & 36 & & & & & & & & & & & & & \\
\hline 5 & $c a$ & 5 & 23 & 19 & 13 & & & & & & & & & & & & \\
\hline 6 & $p g i$ & 7 & 23 & 14 & 23 & 14 & & & & & & & & & & & \\
\hline 7 & $h k-1$ & 9 & 2 & 8 & 21 & 9 & 8 & & & & & & & & & & \\
\hline 8 & $a a t-1$ & - & 10 & 12 & 7 & 2 & 11 & 2 & & & & & & & & & \\
\hline 9 & sucr & 2 & 1 & - & 2 & 3 & 2 & 3 & - & & & & & & & & \\
\hline 10 & $m d h-2$ & 5 & 1 & 3 & 9 & 5 & - & 9 & - & - & & & & & & & \\
\hline 11 & $a d h-1$ & 1 & 0 & 4 & - & - & 1 & 0 & - & - & - & & & & & & \\
\hline 12 & $a d h-2$ & 7 & 1 & 14 & 15 & - & - & - & - & - & - & 1 & & & & & \\
\hline 13 & g6pdh & 4 & 4 & 10 & - & 4 & - & - & - & - & - & - & - & & & & \\
\hline 14 & est -1 & 7 & - & 5 & 7 & - & - & - & - & - & - & - & 8 & - & & & \\
\hline 15 & $h k-2$ & - & 12 & 15 & 14 & - & 6 & - & - & - & - & - & - & - & - & & \\
\hline 16 & acon-2 & - & 11 & 11 & - & 12 & - & - & - & - & - & - & - & 3 & - & - & \\
\hline
\end{tabular}

one recombinant among 92 informative offspring, giving an overall estimate of the male recombination fraction of $r_{m}=0.011$, which is highly significant (lod score $Z=29$ ). This is fully consistent with the result from female-informative families, which showed a zero summed $F R_{\text {min }}$, consistent with synteny.

An additional four locus pairs, each from a single male-informative family, were detected by LINKAGE1. However, calculation of the lod score corresponding to the phase-unknown maximum likelihood estimate of $r_{m}$ showed that the support for linkage was very weak. $A d h-2 / i d h-2$ in cross 2 had $Z=2.7$ $\left(r_{m}=0\right), a d h-2 / m d h-1$ in cross 2 had $Z=0.54$ $\left(r_{m}=0.2\right)$, aat-1/idh-2 in cross 15 had $Z=0.54$ $\left(r_{m}=0.2\right)$, and $m d h-1 / m p i$ in cross 2 had $Z=0.38$ $\left(r_{m}=0.25\right)$. It is curious that three of these involved cross 2, with only 12 offspring. Because none of these locus pairs occurred in any female-informative families, synteny cannot be ruled out with the present data. Only the first of these has a lod score approaching the traditional significance threshold of 3. More data will be required to increase these lod scores and provide convincing support for linkage, or alternatively to provide evidence against synteny from forbidden recombinants in female-informative families.

Of the remaining six male-informative tables detected as significant by LINKAGE-1, five corresponded to locus pairs that were informative in females and showed four or more forbidden recombinants.
The sixth was informative in a single family only, but the deviation from independent assortment could not be accounted for by linkage under either alternative for phase.

\section{Search for allozyme loci on the autosome-W translocation}

The method of forbidden recombinants was also applied to examine allozymes for behaviour characteristic of the autosome involved in the $\mathrm{A}^{\mathrm{w}}$ translocation in females. All 16 autosomal loci heterozygous in one or more mothers showed large numbers (4-80) of forbidden recombinants and so none of them is likely to occur on $A^{w}$. This test was not possible for the three loci that were heterozygous only in males, namely $f h, i d h-2$ and $m d h-1$. However, if the aat-1/idh-2 linkage is valid, idh-2 cannot be involved in the translocation because aat-1 is not, and if the $m d h-1 / m p i$ linkage is valid, $m d h-1$ cannot be involved in the translocation because $m p i$ is not.

\section{Discussion}

Our results confirm and extend previous allozyme studies on $Y$. padellus and other Yponomeuta. We now have evidence that at least one unusual, previously puzzling electromorph results from a nullactivity allele. Our results on sex-linked loci provide 
an interesting comparison to other species of Yponomeuta. Additional results from a few 'anomalous' individuals indicate that gynandromorphy in Yponomeuta, although rare, may be detectable by isozyme analysis. Furthermore, we have tested a number of autosomal loci for their possible involvement in a sex-chromosome translocation. Finally, our analysis shows that isozymes will be a powerful tool in the comparative genomics of the Lepidoptera. We discuss each of these points in turn.

The unusual two-banded phenotype for hydroxybutyrate dehydrogenase had been seen in earlier population surveys of Y. padellus (L. E. L. Raijmann, unpublished data); it was completely absent in some populations, but accounted for up to 10 per cent of the sample in others. The present experiments confirmed the transmission of a null allele in five different crosses. Because of the ability of the null allele product to dimerize with the active product of other alleles, the phenotypes of null heterozygotes were distinguishable from phenotypes of homozygotes for the active allele. Furthermore, two different non-null alleles entering into these crosses interacted with the null allele in this way, enabling estimation of the electrophoretic mobility of the 'invisible' null allele product homodimer.

Single-pair crosses had previously revealed the existence of fudh as a sex-linked locus in Y. cagnagellus (Menken, 1980b). In addition, no fudh heterozygous females have ever been seen in population surveys of $Y$. cagnagellus, $Y$. evonymellus, $Y$. vigintipunctatus, Y. yanagawanus, Y. mahalebellus and $Y$. rorellus (Menken, 1982, 1987 and unpublished data). Sex-linkage of est-2 had also been suggested for $Y$. cagnagellus because of the absence of female heterozygotes (Menken, 1980b). Our experiments confirmed the sex-linked inheritance of both est-2 and fudh in the closely related species Y. padellus. The highly polymorphic 6pgdh, however, had been explicitly rejected as a sex-linked locus in crosses of Y. cagnagellus (Menken, 1980b). This shows that sex-linkage groups in the two closely related species are not completely conserved, and adds another level of complexity in assessing the role of sex-linked loci in reproductive isolation and host-race formation as suggested by Sperling (1994).

Rare individuals, apparently female and heterozygous for sex-linked loci, have also been observed in other studies of Yponomeuta. To investigate the population structure of $Y$. padellus, over 40 different populations and thousands of individuals have been analysed and sometimes heterozygote females have been encountered at 6pgdh (L. E. L. Raijmann, unpublished data). Although simple scoring errors could be at fault, there is a biological phenomenon that could account for a misinterpretation of sex and could therefore explain these results. Kuijten (1973) described a gynandromorph specimen of $Y$. cagnagellus. The (male) right half of the genitalia was normally developed, whereas the left half showed a small and distorted valva and lacked male structures such as the socius and gnathos. On the other hand, female structures such as anal papilla and apophysis (posterior and anterior) could be distinguished at the left side. Because $Y$. cagnagellus and $Y$. padellus are genetically and morphologically closely related, this suggests that the five individuals with exceptional sex-linked genotypes in our study could be gynandromorphs, although the usual sexing did not reveal aberrant patterns. Unfortunately, destruction of the entire body of these small insects to obtain sufficient homogenate for electrophoresis has precluded further direct investigation of that question.

Of the five individuals with exceptional genotypes at sex-linked loci, the two from cross 23 (Table 2) are the most enigmatic. They are both heterozygous at fudh, which would imply that they are males, but they are both homozygous at $6 p g d h$, which would imply that they are females (given their parental genotypes). This means that, irrespective of the morphologically diagnosed sex of these two progeny (male), the two sex-linked loci are in conflict with one another. (Their est-2 genotypes are consistent with either sex.) If these individuals have inherited the maternal $\mathrm{Z}$ chromosome, one would expect expression of maternal alleles at both fudh and $6 p g d h$, yet the latter is not observed in these two individuals. Even if gynandromorphy is caused by chimaerism in which some cell lineages have lost a maternal $\mathrm{Z}$ chromosome, differential expression of fudh and $6 p g d h$ from the maternally derived $\mathrm{Z}$ chromosomes in the other lineages would need to be invoked to explain the observations. Perhaps only a fragment of the maternal $\mathrm{Z}$ was originally transmitted or subsequently lost. Identification of other sex-linked loci and correlation of their expression patterns in putative gynandromorphs would help to address these questions.

A remarkable observation by Sheppard et al. (1985) may be relevant here. One of many crosses they set up to investigate the genetics of mimetic wing patterns in Heliconius butterflies produced two gynandromorphs from a brood of 35 offspring. These two exceptional individuals were also chimaeric for three other wing colour characteristics segregating in the cross. The striking occurrence of completely different phenotypes on the left and right 
side of the same individual suggested that they were double-fertilization 'twins' arising from binucleate eggs. Perhaps the same phenomenon is also responsible for the anomalous individuals in our study. Confirmation of this would require a careful genetic and cytogenetic study in which the rare gynandromorphs would be dissected after identification and isozymes assayed separately in each half. For the purposes of the present linkage study such individuals should be excluded from the analysis because of the confounding of phenotypes resulting when both halves of the 'twin' are homogenized together.

Our attempt to find allozymes located on the autosomal portion of the $\mathrm{A}^{\mathrm{w}}$ translocation ruled out the 16 loci heterozygous in mothers in our dataset. Using a similar approach in pedigree studies of a primitive termite, Santos \& Luykx (1985) found two allozyme loci located on autosomes involved in a multiple translocation system with the sex chromosomes. Alternatively, the method of Berlocher (1993) might be used to look for disequilibrium between allozymes and the sex chromosomes in population samples.

Detection of autosomal linkage in Lepidoptera is made difficult by the large number of chromosomes, but facilitated by the absence of crossing-over in females. The latter is well documented by several cytogenetic studies demonstrating achiasmatic oogenesis in Lepidoptera (including Yponomeuta, Nilsson et al., 1988) as well as genetic studies showing an absence of recombination when the informative parent is female (reviewed in Robinson, 1971). If this is a universal feature of the genetics of Lepidoptera, as seems likely, it will greatly facilitate comparative genomic studies in this order of insects.

Achiasmatic oogenesis, however, complicates the estimation of recombination fractions and their statistical significance. Ideally, maximum likelihood methods would be employed, combining data from interchromosomal recombination in achiasmatic females and inter- and intrachromosomal recombination in males into a single likelihood function. This would enable application of the widely used lod score method (Ott, 1991), in which evidence for linkage is evaluated statistically by computation of the lod score $Z$. However, most computer programs for linkage mapping assume that crossing-over can occur in both sexes, yielding incorrect $Z$-values for Lepidoptera. The method of forbidden recombinants and the lod score method have been simultaneously applied to the case of a single $F_{2}$ cross between two inbred strains, with gametic phase known and two alleles per locus, in the construction of a linkage map for the domesticated silkmoth (Shi et al., 1995). However, in our Y. padellus data gametic phase was unknown because grandparents were not analysed, data were produced from several crosses, the parents were not inbred, not all loci behaved as in an $F_{2}$, and several loci had three or more alleles. Our solution to this problem was to handle the method of forbidden recombinants and the lod score method sequentially, by separately analysing female-informative and male-informative combinations. This approach is conservative for linkage detection, which was desirable because with 31 chromosomes in Y. padellus (Gershenzon, 1967; Nilsson et al., 1988), the prior probability of linkage was very low. For those few cases in which we had to estimate the male recombination fraction $r_{m}$, we could confine our analysis to male-informative matings and use conventional approaches for calculating $Z$.

Our study illustrates how high levels of naturally occurring allozyme polymorphism in Lepidoptera can be utilized by the method of forbidden recombinants to classify loci into mutually asyntenic groups. Even modest family sizes can produce sufficient numbers of forbidden recombinants conclusively to rule out synteny among several locus pairs. The main factor limiting classification of all allozymes into unique synteny groups is the fraction of pairwise combinations that are actually informative in the study. This is affected not only by the levels of polymorphism in the base population, but also by the occurrence of all possible pairs of heterozygotes among the parents. Our dataset, based on a random population sample of parents, provided femaleinformative tests for only about one-third and maleinformative tests for about half of the 171 possible locus pairs among the 19 polymorphic autosomal loci. For univoltine species such as $Y$. padellus, the most efficient way to provide information on the remaining untested locus pairs would be to set up another round of crosses, then screen the parents and only analyse progeny contributing to untested linkages. The simplicity of this approach lends itself well to comparative studies with other species of Yponomeuta. Even beyond this interesting genus, the widespread polymorphism of allozymes in natural populations of most species, and their relative ease and speed of analysis, will make them very useful anchor loci for comparative genomics of the Lepidoptera as a whole.

\section{Acknowledgements}

We wish to thank Coenraad R. Jager and Daniel F. Cleary for comments on an earlier draft of this 
manuscript. Three anonymous reviewers made helpful comments; we thank especially the one who brought the Heliconius gynandromorphs to our attention. Portions of the data analysis were supported by an NSF EPSCoR grant to the State of South Carolina.

\section{References}

ARDUINO, P. AND BULLINI, L. 1985. Reproductive isolation and genetic divergence between the small ermine moths Yponomeuta padellus and Yponomeuta malinellus. Atti. Acad. Naz. Lincei. Cl. Sci. Fis. Mat. Nat. Rend., 18, $33-61$.

AYALA, F. J., POWELl, J. R., TRACEY, M. L., MOURĀO, C. A. AND PÉREZ-SALAS, S. 1972. Enzyme variability in the Drosophila willistoni group. IV. Genic variation in natural populations of Drosophila willistoni. Genetics, 70, 113-139.

BENNETZEN, J. L. AND FREELING, M. 1993. Grasses as a single genetic system: genome composition, colinearity and compatibility. Trends Genet., 9, 259-261.

BERLOCHER, S. H. 1993. Gametic disequilibrium between allozyme loci and sex chromosomes in the genus Rhagoletis. J. Hered., 84, 431-437.

CLAYTON, J. W. AND TRETIAK, D. N. 1972. Amine-citrate buffers for $\mathrm{pH}$ control in starch gel electrophoresis. $J$. Fish. Res. Board Canada, 29, 1169-1172.

GERSHENZON, z. s. 1967. (On the question of species independence of the apple, fruit and willow ermine moths.) (In Russian). Vestn. Zool., 3, 38-40.

HARRIS, H. AND HOPKINSON, D. A. 1976. Handbook of Enzyme Electrophoresis in Human Genetics. NorthHolland Publishing Company, Amsterdam.

HEBERT, P. D. N. AND BEATON, M. J. 1989. Methodologies for Allozyme Analysis Using Cellulose Acetate Electrophoresis. Helena Laboratories, Beaumont, TX.

HECKEL, D. G. 1993. Comparative genetic linkage mapping in insects. Ann. Rev. Ent., 38, 381-408.

HENDRIKSE, A. 1988. Hybridization and sex-pheromone responses among members of the Yponomeuta padelluscomplex. Entomologia exp. appl., 48, 213-233.

KUIJTEN, P. J. 1973. A gynandromorph of the small ermine moth, Yponomeuta cagnagellus (Hb.) (Lepidoptera, Yponomeutidae). Ent. Ber. Amst., 33, 221-222.

MENKEN, S. B. J. 1980a. Inheritance of allozymes in Yponomeuta (Lepidoptera). II. Interspecific crosses within the padellus-complex and reproductive isolation. Proc. Kon. Ned. Akad. Wet. (C), 83, 425-431.

MENKEN, S. B. J. 1980b. Inheritance of allozymes in Yponomeuta (Lepidoptera). I. Intraspecific crosses in $Y$. cagnagellus. Proc. Kon. Ned. Akad. Wet. (C), 83, 417-423.

MENKEN, S. B. J. 1982. Biochemical genetics and systematics of small ermine moths (Lepidoptera, Yponomeutidae). Z. Zool. Syst. Evolut-forsch., 20, 131-143.
MENKEN, S. B. J. 1987. Is the extremely low heterozygosity level in Yponomeuta rorellus caused by bottlenecks? Evolution, 41, 630-637.

MENKEN, S. B. J., HERREBOUT, w. M. AND WIEBES, J. T. 1992. Small ermine moths (Yponomeuta): their host relations and evolution. Ann. Rev. Ent., 37, 41-66.

NILSSON, N.-O., LÖFSTEDT, C. AND DÄRVING, L. 1988. Unusual sex chromosome inheritance in six species of small ermine moths (Yponomeuta, Yponomeutidae, Lepidoptera). Hereditas, 108, 259-265.

o'brien, s. J., SEUANEZ, H. N. AND WOMACK, J. E. 1988. Mammalian genome organization: an evolutionary view. Ann. Rev. Genet., 22, 323-351.

отт, J. 1991. Analysis of Human Genetic Linkage (revised edn). Johns Hopkins University Press, Baltimore.

RAIJMANN, L. E. L. 1996. In search for Speciation: Genetical Differentiation and Host Race Formation in Yponomeuta padellus (Lepidoptera, Yponomeutidae). $\mathrm{PhD}$ thesis, University of Amsterdam.

RAIJMANN, L. E. L. AND MENKEN, S. B. J. 1992. Population genetical evidence for host-race formation in Yponomeuta padellus. In: Menken, S. B. J., Visser, J. H. \& Harrewijn, P (eds). Proceedings of the 8th International Symposium on Insect-Plant Relationships, pp. 209-212. Kluwer Academic Publishers, Dordrecht.

Robinson, R. 1971. Lepidoptera Genetics. Pergamon Press, Oxford.

SANTOS, O. AND LUYKX, P. 1985. Holozygosity for sex-linked genes in males of the termite Incisitermes schwarzi. Biochem. Genet., 23, 729-740.

SCHWARTZ, M. K., NISSELBAUM, J. S. AND BODANSKY, O. 1963. Procedure for staining zones of activity of glutamic oxaloacetic transaminase following electrophoresis with starch gels. Am. J. Clin. Pathol., 40, 103-106.

SELANDER, R. K., SMITH, M. H., YANG, S. Y., JOHNSON, W. E. AND GENTRY, J. B. 1971. Biochemical polymorphism and systematics in the genus Peromyscus. I. Variation in the old-field mouse (Peromyscus polionotus). In: Studies in Genetics, vol. 6, pp. 49-90. University of Texas Publication 7013.

SHEPPARD, P. M., TURNER, J. R. G., BROWN, K. S., BENSON, W. W. AND SINGER, M. C. 1985. Genetics and the evolution of Muellerian mimicry in Heliconius butterflies. Phil. Trans. R. Soc. B, 308, 433-610.

SHI, J., HECKEL, D. G. AND GOLDSMITH, M. R. 1995. A genetic linkage map for the domesticated silkworm, Bombyx mori, based on restriction fragment length polymorphisms. Genet. Res., 66, 109-126.

SPERLING, F. 1994. Sex-linked genes and species differences in Lepidoptera. Can. Entomol., 126, 807-818.

SUITER, K. A., WENDEL, J. F. AND CASE, J. S. 1983. LINKAGE1: a computer program for the detection and analysis of linkage. J. Hered., 74, 203-204.

VAN DRONGELEN, W. AND VAN LOON, F. F. A. 1980. Inheritance of gustatory sensitivity in F1 progeny of crosses between Yponomeuta cagnagellus and $Y$. malinellus (Lepidoptera). Entomologia exp. appl., 24, 199-203. 